\title{
Bone necrosis associated with the use of bisphosphonates: Case report
}

\section{Martwica kości żuchwy po zastosowaniu bifosfonianu - opis przypadku}

\author{
Tomasz Krzysztof Jachewicz ${ }^{A-E}$, Joanna Jakiee ${ }^{A, B, D}$ \\ Chair and Department of Oral Surgery, Medical University of Lublin, Lublin, Poland \\ A - research concept and design; $\mathrm{B}$ - collection and/or assembly of data; $\mathrm{C}$ - data analysis and interpretation; \\ $D$ - writing the article; $E$ - critical revision of the article; $F$ - final approval of article
}

Address for correspondence

Tomasz Krzysztof Jachewicz

E-mail: jaho112@op.pl

\section{Funding sources}

none declared

Conflict of interest

none declared

Received on February 22, 2017

Revised on May 03, 2017

Accepted on June 15, 2017

\begin{abstract}
The aim of this study was to present the case of a 68-year-old female patient with jaw bone necrosis associated with the use of bisphosphonates. The case was qualified for the second degree of severity (BRONJ). The study demonstrates a multi-month observation of the patient and describes the sequestrectomy procedure performed, which was supplemented by covering the bone tissue with a collagen sponge. The treatment applied was slightly different from the accepted algorithms of proceedings. The difference was connected with the use of a collagen sponge containing gentamicin (used to cover the bone's surface) after the sequestrectomy. The collagen material, in contrast to the exposed dead bone, is a surface which may be susceptible to epithelization. This gives a possibility to the secondary closing of the wound as a result of granulation and epithelization without the bone's re-exposure. Moreover, the study characterizes indications for the use of bisphosphonates, their chemical structure and mechanism of action. It shows the definition of BRONJ (bisphosphonate-related osteonecrosis of the jaw) and risk factors for its occurrence, as well as prevention and treatment methods.
\end{abstract}

Key words: osteonecrosis, diphosphonates, bisphosphonate related osteonecrosis of the jaw

Słowa kluczowe: martwica kości, difosfoniany, martwica kości żuchwy spowodowana zastosowaniem bisfosfonianu

DOI

10.17219/dmp/74884

Copyright

() 2017 by Wroclaw Medical University

and Polish Dental Society

This is an article distributed under the terms of the

Creative Commons Attribution Non-Commercial License

(http://creativecommons.org/licenses/by-nc-nd/4.0/) 
Therapy with the use of bisphosphonates (BP) is now frequently implemented in the case of oncological, endocrine or orthopedic diseases. ${ }^{1}$ Through the mechanism of action consisting of inhibition of osteoplastic bone tissue resorption, these drugs are used in diseases connected with the skeletal system, e.g. osteoporosis, Paget's disease, multiple myeloma, osteogenesis imperfecta and hypercalcemia associated with malignant tumor. They are often used in the prevention of cancer metastases to the skeletal system. ${ }^{2}$

Bisphosphonates are synthetic analogues of pyrophosphates - i.e. natural molecules occurring in the skeletal system. $^{3-5}$ The high affinity to bones is connected with the presence of a hydroxyl group in their structure. This group is fixed with calcium ions on the surface of hydroxyapatite. ${ }^{6}$ This is followed by incorporation into osteoclasts, leading to their inactivation and finally to their apoptosis. ${ }^{2,7}$ These drugs probably reduce the amount of VEGF protein (a strong proangiogenic factor), lead to a handicap in the formation of new blood vessels and indirectly have a negative impact on bone remodeling. The beneficial impact on the primary disease (by inhibiting the proliferation of tumor cells) and stimulation of their apoptosis were demonstrated among oncological patients. Thanks to the reduced invasiveness and adhesion of tumor cells, the formation of metastases is inhibited. ${ }^{2,8}$

Medicines from this group have been used for more than 40 years. In the vast majority of cases, they improve quality of patients' life and they are well tolerated. One of the more common complications is osteonecrosis of the jaw bone. It is known under the name "bisphosphonate related osteonecrosis of the jaw (BRONJ)".,10 For diagnosis of this disease, 3 elements should be present - the patient received or receives drugs from the BP group, exposure of dead bone tissue in the upper or lower jaw was observed (longer than 8 weeks), and the patient was not treated with the use of radiotherapy in the area of the head and neck. ${ }^{11}$ In 2014, AAOMS (The American Association of Oral and Maxillofacial Surgeons) recommended the change of this term to "medication-related osteonecrosis of the jaw (MRONJ)". ${ }^{12}$ Osteonecrosis associated with the use of bisphosphonates can occur intrinsically, but usually is connected with invasive dental procedures, i.e. tooth extraction (75-86\% of cases). Interestingly, it is more common among patients with cancer (about $0.7-6.7 \%$ ) than in patients treated for osteoporosis $(0.04-0.2 \%) .{ }^{11,12}$ This disease more often affects the lower jaw than the maxilla. Risk factors mainly include: injuries associated with treatment in dental surgery, use of mismatched prosthetic restorations, presence of bone exostosis, odontogenic inflammations, poor oral hygiene, coexisting diabetes, simultaneous chemotherapy and/or steroid treatment, malnutrition and smoking. ${ }^{13-15}$ The route of $\mathrm{BP}$ administration has a great impact. The risk of BRONJ is greater for intravenous therapy (0.8-15\%). Additionally, changes occur faster - about 1 year after the initiation of the therapy. For the oral route, the risk of ne- crosis amounts to $0.01-0.3 \%$, and changes occur not earlier than after 3 years after first oral administration. Necrosis is more often observed after intravenous application of zoledronic acid, pamidronate sodium and alendronate sodium. The occurrence of BRONJ is influenced by the application time of BP, which is different and ranges from 1.5 to 5 years. ${ }^{16,17}$

The mechanism of BRONJ's formation is not well explored. It is believed that bisphosphonates have a negative impact on osteoclasts and they impair bone turnover and bone remodeling, particularly important after tooth extraction. Furthermore, the exposure of bone tissue on the flora of the mouth seems to the unfavorable. It has been confirmed that biofilm presents a higher adhesion to bone saturated with BP than to unchanged bone. These medicines demonstrate toxicity to the soft tissues. This impairs granulation and epithelialization in the area of the alveolus, and this significantly increases the time of exposure to the oral environment. Impairment of angiogenesis also plays a role in the etiology. As a result, proper healing of alveolar tissues does not take place, as well as exposure and osteonecrosis. ${ }^{7}$

The clinical picture often notes the exposure of the bone over a large area. The visible tissue may have a characteristic gray color, there is pain caused by palpation and spontaneous pain, swelling of the surrounding soft tissues, and sometimes pathological fractures can be observed. This study describes the cases of osteonecrosis in the course of BRONJ, where changed hard tissues are invisible and covered with a mucous membrane. In such cases, pain, soft tissue swelling and distension of bones are also noted. Oroantral fistulas and associated inflammation of the maxillary sinus are rarely observed in the case of necrosis. ${ }^{18}$ The radiological image is not characteristic and it resembles the classic inflammation of bone tissue with the formation of necrosis. A histopathological examination notes the necrosis of bone tissue, the change of its architectonics with visible resorption areas, the reduction in the number of blood vessels and fibrosis. Moreover, inflammatory infiltration and bacterial biofilm are visible. ${ }^{19}$

BRONJ should be differentiated taking into account the presence of: dry socket, alveolitis purulenta, maxillary sinusitis, gingivitis, periodontitis, inflammation of periapical tissues and disorders in the temporomandibular joint. ${ }^{7}$

\section{Case report}

A 68-year-old female patient came to the Department of Oral Surgery at the Medical University of Lublin for the sanitation of the oral cavity. In the medical history, the patient stated that for 10 years she had had oncological treatments because of left breast cancer with metastases to the lungs. Combination therapy was connected with the performance of mastectomy and adjuvant chemotherapy. The patient had taken 3 cycles of chemotherapy. The last cycle had been received 3 months prior to the visit in 


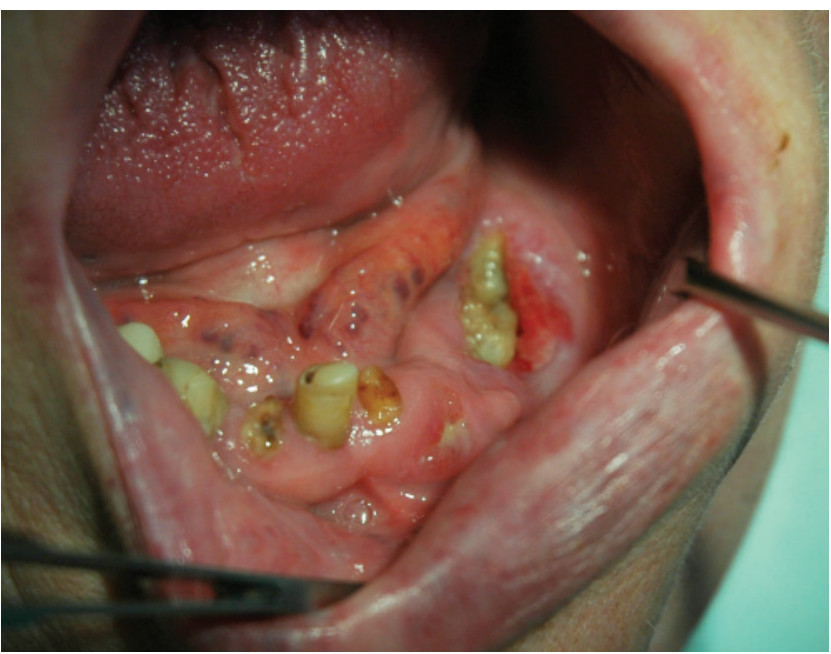

Fig. 1. Intraoral view

the dental office. Carboplatin plus gemcitabine, as well as zoledronic acid were applied with the use of intravenous infusion. Currently, the patient was under constant observation of the oncologist and she was having hormonal treatment. The intraoral examination found the residual roots of teeth 35 and 34. It was decided to extract the above-mentioned teeth in an outpatient procedure. One hour before treatment, the patient got $2 \mathrm{~g}$ of amoxicillin orally, and then teeth 35 and 34 were removed under local anesthesia ( $2 \%$ of lidocaine with noradrenaline). It was recommended to continue the antibiotic therapy $-2 \mathrm{~g}$ of amoxicillin per day in 2 divided doses for 7 days. The post-treatment period was uneventful.

After 6 months, the patient came back in order to remove fractured teeth 32 and 33. Just like in the first time, the procedure was carried out under local anesthesia $(2 \%$ of lidocaine with noradrenaline) and antibiotic prophylaxis ( $2 \mathrm{~g}$ of amoxicillin $1 \mathrm{~h}$ before surgery). Dosage of $2 \mathrm{~g}$ of amoxicillin per day in 2 divided doses for 7 days was prescribed. After 7 days, the sutures were removed and the proper healing of the wounds was noted.

After a further 6 months, the patient returned in order to report pain in the area of the extracted teeth 32-35. An extraoral study presented enlarged, hard subman-

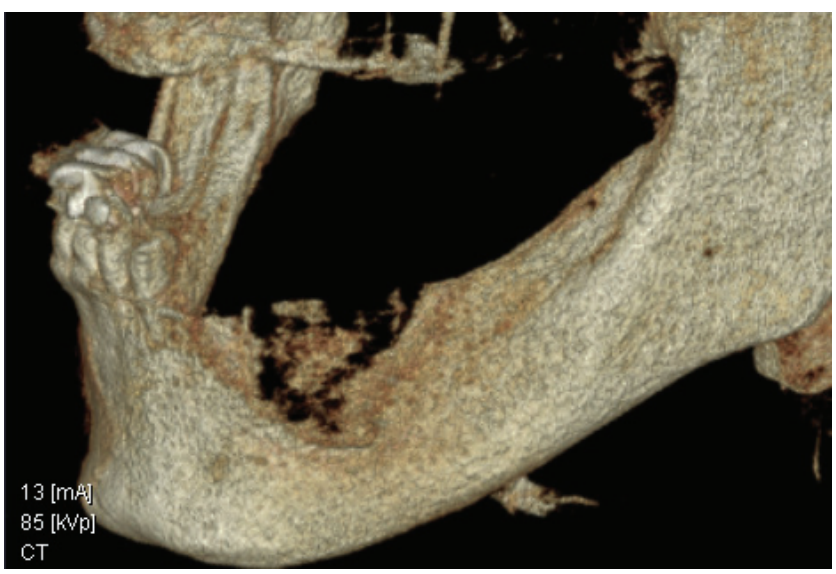

Fig. 2. 3D reconstruction of the mandible dibular lymph nodes (group B) which were very painful on palpation. Intraorally, at the tip of the alveolar part of the mandible in an area of about $2 \mathrm{~cm}$, exposed bone tissue was visible. Below there were numerous fistulas, and more broken roots of teeth 31 and 42 . Furthermore, the $3^{\text {rd }}$-degree mobility of tooth 42 was observed (Fig. 1). What is more, dressing wound was carried out and antibiotic therapy was prescribed - clindamycin $300 \mathrm{mg}$ once every $8 \mathrm{~h}$. The patient's CBCT showed a sequestrum in the area of the removed teeth, 32-35 (Fig. 2-5).

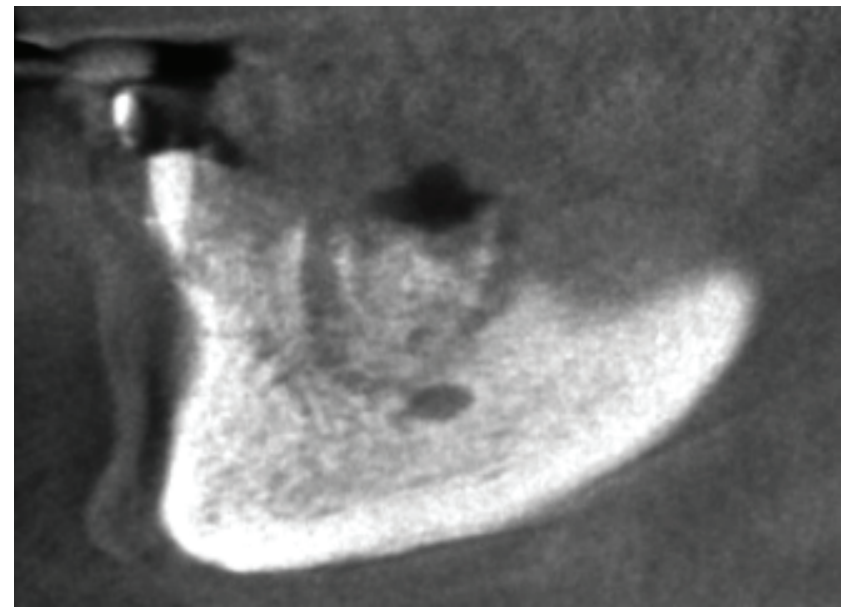

Fig. 3. Sagittal projection of mandible, with visible sequestrum

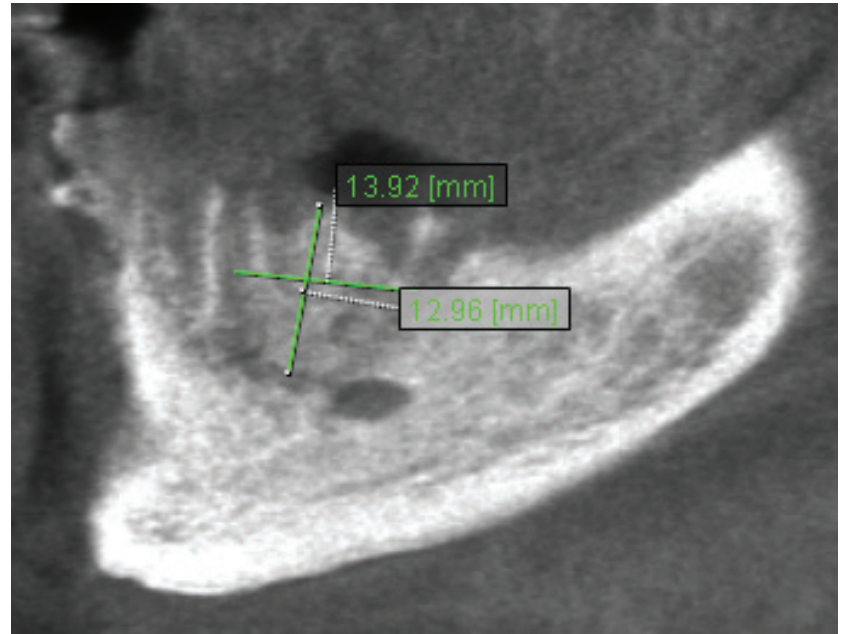

Fig. 4. Sagittal projection of mandible, with visible dimension of sequestrum

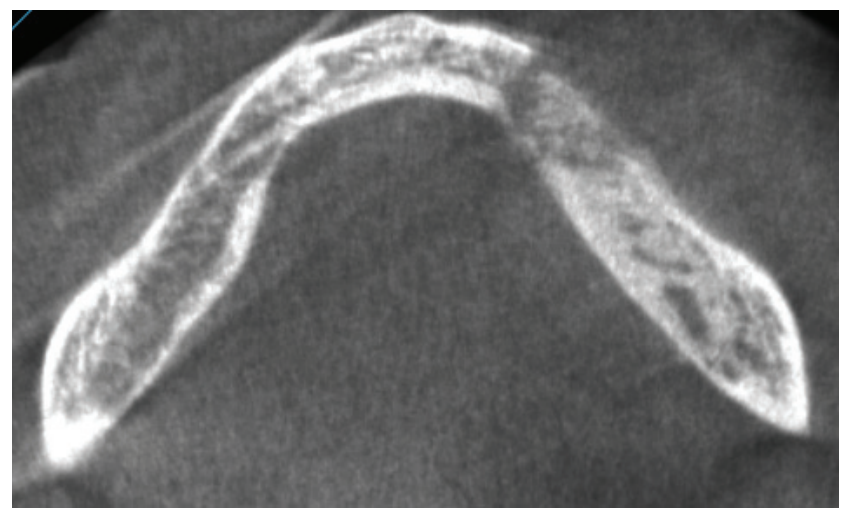

Fig. 5. Horizontal projection of the mandible 


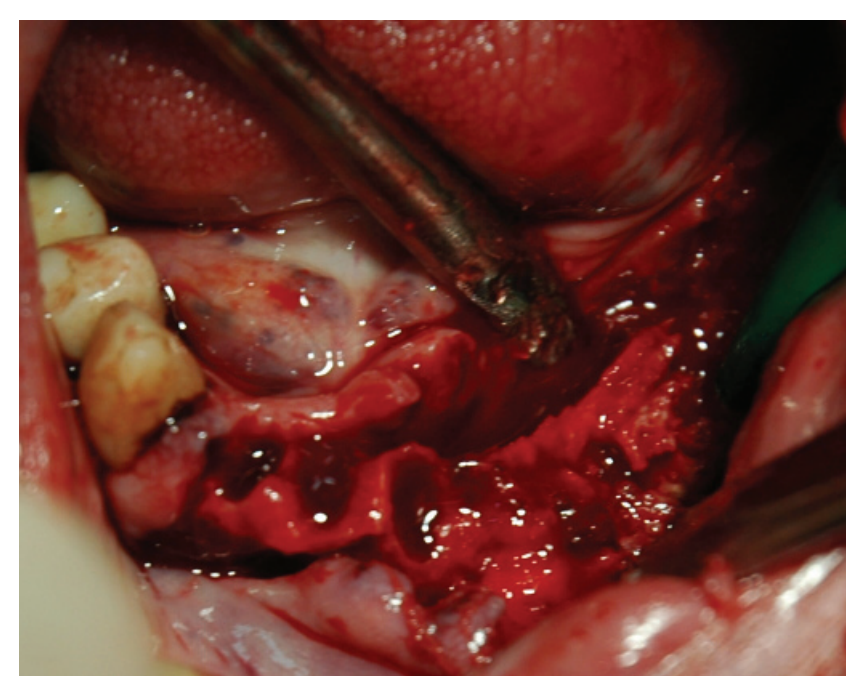

Fig. 6. Intraoperative view with visible sequestrum

After a period of 5 days from the beginning of the antibiotic therapy and after consultation with the patient's oncologist, the next treatment was carried out. Under alveolar, lingual and mental nerve block anesthesia (4\% articaine with adrenaline), teeth 31,41 and 42 were removed, a triangular mucoperiosteal flap was created, the mental nerve was revealed and the sequestrum was cut out with the use of a piezosurgery unit (Fig. 6). Plasticity of the bone tissue was performed. The wound was rinsed with ozone water, and a collagen sponge with gentamicin $5 \times 5 \mathrm{~cm}$ was placed. The wound was closed by non-absorbable single sutures 4.0 (Fig. 7).

The removed material was sent for histopathological examination. The following result from this examination was obtained: "Fragments of bone tissue in purulent inflammation with areas of necrosis". The post-treatment period was uneventful. The patient had regular follow-up visits and her sutures were removed after 14 days. On the $3^{\text {th }}$ day after the surgery, clindamycin was discontinued and the patient started to take doxycycline (100 mg per

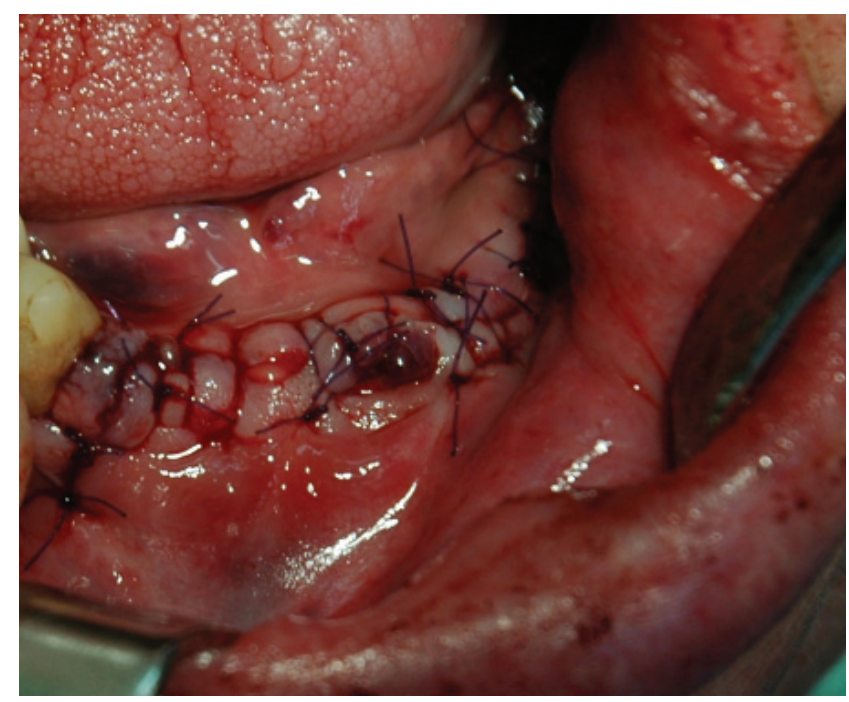

Fig. 7. Wound after suturing

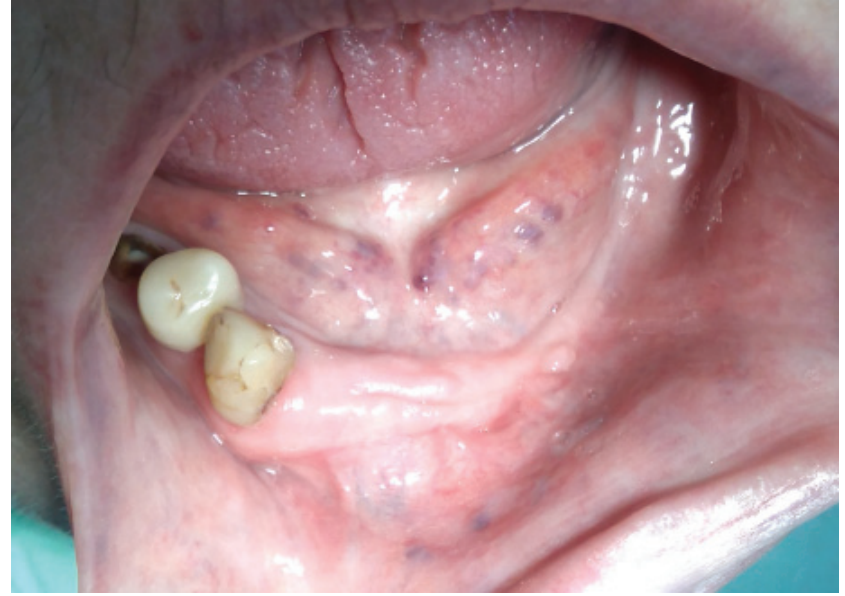

Fig. 8. Intraoral view 3 months after surgery

day in one dose for a period of 3 months). Three months after the surgery, a properly healed mucous membrane without any signs of inflammation was noted (Fig. 8).

\section{Discussion}

The majority of authors recommend the preparation of a patient for BP treatment covering sanitation of the oral cavity. ${ }^{20}$ If it is necessary to perform tooth extraction, the beginning of the BP therapy should be postponed (up to 2-3 weeks), until the healing of post-extraction wounds. When the patient is already receiving BP, especially via the intravenous route, dental procedures should be limited to conservative, endodontic and non-surgical periodontal treatments. Invasive treatments are not recommended. ${ }^{1}$

Patients who are in the at-risk group should be informed about the possibility of developing BRONJ. They require regular control visits to check the state of the oral cavity, as well as preventive and conservative treatment. ${ }^{21}$

The algorithm for patients with diagnosed BRONJ depends on the severity of the disease. There are 3 degrees of severity. In the $1^{\text {st }}$ degree, there is an exposed and dead, but asymptomatic bone fragment without signs of infection. The procedure consists of conservative treatment using rinses of $0.12-0.2 \%$ of chlorhexidine. Surgical treatment is not recommended. In the $2^{\text {nd }}$ degree, there is an exposed and dead bone fragment, which is accompanied by pain and clinical symptoms of infection. Conservative treatment with the use of rinses of $0.12-0.2 \%$ chlorhexidine and long-term antibiotic therapy is applied. In the case of the $3^{\text {rd }}$ degree, apart from the exposed necrotic bone fragment, pain and infection features, there is also pathological fracture or intraoral fistula. Surgical treatment along with antibiotic therapy and antiseptic local treatment is recommended. Regardless of the severity of the disease, the removal of bone necrosis is always necessary. In the case of pathological fractures, segmental resection and reconstruction of the affected bone should be carried out. Furthermore, fistulas ought to be surgically removed. ${ }^{16}$ 
In cases where there is a need to perform surgical treatment in the oral cavity (during BP treatment), it is recommended to carry out the hygienization and oral hygiene instructions. A few days before the surgery, antiseptic mouthwashes on the basis of $0.12-0.2 \%$ chlorhexidine should be applied. Antibiotic therapy ought to be implemented. Formulations typical for the maxillo-facial area (amoxicillin, clindamycin, doxycycline) are applied in typical doses. It is recommended to start the antibiotic therapy 1-3 days before the surgery. Medications should be administered in the long term - from 3 months to over a year. ${ }^{22}$

Each treatment should be performed in the least invasive way. The resulting wounds ought to be initially closed and the doctor should avoid the mobilization of large flaps of soft tissues. Such a procedure reduces the exposure of bone tissue to the flora of the oral cavity, which in turn supports the healing process without complications. ${ }^{1}$

After the completion of a necrectomy, some authors suggest the covering of bone with platelet-rich plasma, which stimulates osteoblasts, accelerates epithelialization, reduces inflammation and stimulates angiogenesis. ${ }^{23}$ A good result was obtained after the application of ozone combined with surgical treatments and antibiotic therapy. ${ }^{24}$ Moreover, research concerning the use of hyperbaric oxygen therapy especially in combination therapy is being carried out, and the results are promising. ${ }^{25}$ The procedure (carried out in the above-described way) has very good effects in the case of patients who take BP orally. More than $90 \%$ of patients are cured. In the case of intravenous therapy, the effectiveness of treatment is worse, only $50-88 \% .{ }^{17}$

More and more patients qualify for therapy with the use of BP. Importantly, patients should be appropriately prepared and dental treatment ought to be carried out in accordance with the applicable regulations. If there are complications in the form of BRONJ, patients should be directed to clinical hospitals with adequate algorithms for procedures and experience in such patients.

The case of the female patient described in this article qualified for the $2^{\text {nd }}$ degree of severity (BRONJ). The treatment applied was slightly different from the accepted algorithms of procedures. The difference was connected with the use of a collagen sponge containing gentamicin (used to cover the bone's surface) after the sequestrectomy. It was used for 2 reasons. First of all, the authors' experience shows that the main problem during the healing period is the dehiscence of edges in the originally closed wound. The collagen material, in contrast to the exposed dead bone, is a surface which may be susceptible to epithelization. This gives a possibility to the secondary closing of the wound as a result of granulation and epithelization without the bone's re-exposure. Additionally, many studies confirm the presence of plentiful bacterial colonies in the area of sequestrum. Gentamicin (released for several days from the collagen carrier) demonstrates a proper spectrum and it is directly applied to the location of the inflammation. The use of a collagen sponge with gentamicin significantly improves the healing of bone tissue, increasing the chances of the patient's recovery.

\section{References}

1. Patel V, McLeod NM, Rogers SN, Brennan PA. Bisphosphonate osteonecrosis of the jaw - a literature review of UK policies versus international policies on bisphosphonates, risk factors and prevention. Br J Oral Maxillofac Surg. 2011;49:251-257.

2. Van Acker HH, Anguille S, Willemen Y, Smits EL, Van Tendeloo VF. Bisphosphonates for cancer treatment: mechanisms of action and lessons from clinical trials. Pharmacol Ther. 2016;158:24-40.

3. Russell RG, Croucher PI, Rogers MJ. Bisphosphonates: Pharmacology, mechanisms of action and clinical uses. Osteoporos Int. 1999;9:66-80.

4. Coleman RE, McCloskey EV. Bisphosphonates in oncology. Bone, 2011;49:71-76.

5. Nisi M, La Ferla F, Karapetsa D, et al. Risk factors influencing BRONJ staging in patients receiving intravenous bisphosphonates: A multivariate analysis. Int J Oral Maxillofac Surg. 2015;44:586-591.

6. Sewerynek E, Stuss M. Bisphosphonates - antiresorptive drugs with analgesic effect? Przegląd Menopauz. 2007;5:276-282 [in Polish].

7. Boff RC, Salum FG, Figueiredo MA, Cherubini K. Important aspects regarding the role of microorganisms in bisphosphonate-related osteonecrosis of the jaws. Arch Oral Biol. 2014:59:790-799.

8. Voss PJ, Joshi Oshero J, Kovalova-Müller A, et al. Surgical treatment of bisphosphonate-associated osteonecrosis of the jaw: Technical report and follow up of 21 patients. J Craniomaxillofac Surg. 2012;40:719-725.

9. Jakiel J, Rahnama M, Szczerba-Gwóźdź J. Treatment of bisphosphonate-related osteonecrosis of the jaws - a report of seven cases. Contemp Oncol. 2016;20:486-490.

10. López-Cedrún JL, Sanromán JF, Garcia A, et al. Oral bisphosphonate-related osteonecrosis of the jaws in dental implant patients: A case series. Br J Oral Maxillofac Surg. 2013;51:874-879.

11. Qaisi M, Hargett J, Loeb M, Brown J, Caloss R. Denosumab related osteonecrosis of the jaw with spontaneous necrosis of the soft palate: Report of a life threatening case. Case Rep Dent. 2016;ID5070187.

12. Morita H, Imai Y, Yoneda M, Hirofuji T. Applying orthodontic tooth extrusion in a patient treated with bisphosphonate and irradiation: A case report. Spec Care Dent. 2017;37:43-46.

13. Drozdowska B. Osteonecrosis of the jaw. Pol J Endocrin. 2011;62:88-92 [in Polish].

14. Ikeda T, Kuraguchi J, Kogashiwa Y, Yokoi H, Satomi T, Kohno N. Successful treatment of bisphosphonate-related osteonecrosis of the jaw (BRONJ) patients with sitafloxacin: New strategies for the treatment of BRONJ. Bone, 2015;73:217-222.

15. Otto S, Schreyer C, Hafner S, et al. Bisphosphonate-related osteonecrosis of the jaws: Characteristics, risk factors, clinical features, localization and impact on oncological treatment. J Craniomaxillofac Surg. 2012;40:303-309.

16. Shintani T, Hayashido $\mathrm{Y}$, Mukasa $\mathrm{H}$, et al. Comparison of the prognosis of bisphosphonate-related osteonecrosis of the jaw caused by oral and intravenous bisphosphonates. Int J Oral Maxillofac Surg. 2015;44:840-844.

17. Jakiel J, Orzędała-Koszel U. Prevention and treatment of bisphosphonate-related osteonecrosis of the jaw - a review of the literature. Twój Przeglad Stomatol. 2016;11:30-34 [in Polish].

18. Mast $G$, Otto $S$, Mücke $T$, et al. Incidence of maxillary sinusitis and oro-antral fistulae in bisphosphonate-related osteonecrosis of the jaw. J Craniomaxillofac Surg. 2012;40:568-571.

19. Reich W, Bilkenroth U, Schubert J, Wickenhauser C, Eckert AW. Surgical treatment of bisphosphonate-associated osteonecrosis: Prognostic score and long-term results. J Craniomaxillofac Surg. 2015;43:1809-1822.

20. Silva PG, Ferreira Junior $A E$, Teófilo $C R$, et al. Effect of different doses of zoledronic acid in establishing of bisphosphonate-related osteonecrosis. Arch Oral Biol. 2015;60:1237-1245.

21. Bauer JS, Beck N, Kiefer J, Stockmann P, Wichmann M, Eitner S. Awareness and education of patients receiving bisphosphonates. J Craniomaxillofac Surg. 2012;40:277-282. 
22. Rupel K, Ottaviani G, Gobbo M, et al. A systematic review of therapeutical approaches in bisphosphonates-related osteonecrosis of the jaw (BRONJ). Oral Oncol. 2014;50:1049-1057.

23. Tsai LL, Huang YF, Chang YC. Treatment of bisphosphonate-related osteonecrosis of the jaw with platelet-rich fibrin. J Formos Med Assoc. 2015;115:585-586.

24. Fliefel R, Tröltzsch M, Kühnisch J, Ehrenfeld M, Otto S. Treatment strategies and outcomes of bisphosphonate-related osteonecrosis of the jaw (BRONJ) with characterization of patients: A systematic review. Int J Oral Maxillofac Surg. 2015;44:568-585.

25. Freiberger JJ, Padilla-Burgos R., McGraw T, et al. What is the role of hyperbaric oxygen in the management of bisphosphonate-related osteonecrosis of the jaw: a randomized controlled trial of hyperbaric oxygen as an adjunct to surgery and antibiotics. J Oral Maxillofac Surg. 2012;70:1573-1583. 